\title{
Characteristics of Metachronous Gastric Tumors after Endoscopic Submucosal Dissection for Gastric Intraepithelial Neoplasms
}

\author{
Tomoyuki Boda, ${ }^{1}$ Masanori Ito, ${ }^{1}$ Shiro Oka, ${ }^{2}$ Yoko Kitamura, ${ }^{1}$ \\ Norifumi Numata, ${ }^{1}$ Yoji Sanomura, ${ }^{2}$ Taiji Matsuo, ${ }^{2}$ Shinji Tanaka, \\ Masaharu Yoshihara, ${ }^{3}$ Koji Arihiro, ${ }^{4}$ and Kazuaki Chayama ${ }^{1}$ \\ ${ }^{1}$ Department of Gastroenterology and Metabolism, Hiroshima University, 1-2-3 Kasumi, Minami-Ku, Hiroshima 734-8551, Japan \\ ${ }^{2}$ Department of Endoscopy, Hiroshima University Hospital, 1-2-3 Kasumi, Minami-Ku, Hiroshima 734-8551, Japan \\ ${ }^{3}$ Health Service Center, Hiroshima University, 1-7-1 Kagamiyama, Higashi-Hiroshima 739-8514, Japan \\ ${ }^{4}$ Department of Pathology, Hiroshima University Hospital, 1-2-3 Kasumi, Minami-Ku, Hiroshima 734-8551, Japan \\ Correspondence should be addressed to Shiro Oka; oka4683@hiroshima-u.ac.jp
}

Received 2 December 2013; Revised 3 January 2014; Accepted 4 January 2014; Published 11 February 2014

Academic Editor: Sergio Morini

Copyright (C) 2014 Tomoyuki Boda et al. This is an open access article distributed under the Creative Commons Attribution License, which permits unrestricted use, distribution, and reproduction in any medium, provided the original work is properly cited.

Background. Recently, endoscopic submucosal dissection (ESD) has become a standard treatment method for early gastric cancer and concurrent stomach preservation. However, metachronous recurrences have become a major problem. We evaluated the incidence and clinicopathologic features of and examined the risk factors for metachronous gastric tumors. Methods. A total of 357 patients who underwent ESD for gastric tumors (245 early gastric cancers and 112 adenomas) and were followed up for more than 12 months without recurrence within the first 12 months were enrolled. We investigated the incidence and clinicopathologic features of metachronous tumors after ESD. We also analyzed the potential risk factors for metachronous tumors using the KaplanMeier method and Cox's proportional hazards model. Results. The annual incidence of metachronous tumors after ESD was $2.4 \%$. The median period until discovery after initial ESD was 26.0 months, and the median observation period was 52.6 months. Male patients developed metachronous tumors more frequently $(P=0.04)$, and the hazard ratio of female to male patients was 0.36 (95\% confidence interval: 0.11-0.89). Conclusions. Patients with a previous history of gastric tumors have a high risk of subsequent gastric tumor development and male patients should be carefully followed up after ESD for gastric tumor.

\section{Introduction}

Gastric cancer is the second most frequent cause of cancer death, and the incidence of gastric cancer among developed countries is the highest in Japan [1]. A number of epidemiological studies have indicated that Helicobacter pylori (H. pylori) infection is significantly related to gastric cancer development [2-4]. Approximately $10-20 \%$ of gastric cancer patients develop multiple synchronous and metachronous cancers [5-8]. To detect early gastric cancer (EGC) after treatment, surveillance procedures should be carefully adhered to.

In recent years, endoscopic submucosal dissection (ESD) for EGC has been widely performed in Japan. With this method, stomach preservation and maintenance of the patients' quality of life are possible [9-12]. However, this approach has been associated with an increase in the risk of gastric cancer recurrence, especially metachronous multiple cancers. The cumulative 3-year incidence of metachronous multiple gastric cancer after partial gastrectomy for EGC was reported to be $1.9 \%$ [13]. Previous studies also reported that the annual incidence of metachronous multiple gastric cancer after ESD for EGC was 2.6-3.5\% [7, 14, 15]. However, the median observation periods of these studies are short (less than 3 years) and there is no study using gastric tumor including adenoma, which generally becomes indication for ESD in Japan, because the pathological finding after ESD occasionally shows adenocarcinoma even though preoperative biopsy showed adenoma [16]. 
In the present study, we evaluated the incidence and clinicopathologic features of metachronous multiple tumors that developed during long-term observation and investigated whether we could predict the occurrence of such tumors on the basis of the patient and tumor features during initial ESD.

\section{Materials and Methods}

2.1. Patients. We enrolled 1,087 consecutive patients with gastric tumors (766 EGCs and 321 adenomas) who underwent ESD at Hiroshima University Hospital between April 2002 and May 2010. We excluded patients with 28 previous gastric surgical histories, 11 local gastric tumor recurrences, 6 gastric mucosa-associated lymphoid tissue lymphoma, 3 Barrett's adenocarcinoma, and 611 patients who had not been followed-up for more than 12 months. Sixty patients who underwent gastric surgery after ESD and 11 patients who underwent $H$. pylori eradication therapy were also excluded. A final total of 357 patients (273 male, 84 female; mean age: 67.4 years) were enrolled in this study, including 245 EGC patients and 112 adenoma patients. Three hundred and thirtyfive patients (94\%) were resected as curative resection according to the Japanese gastric cancer treatment guidelines [17] and others were observed without additional surgical resection. The median observation period was 52.6 months (range: 12.2-113.4 months). Three hundred and twelve patients (88\%) were followed up by annual endoscopic examination in our hospital. We defined a metachronous tumor as a new tumor that developed in another region at least 12 months after ESD.

The protocol was approved by the Ethics Committee of Hiroshima University Hospital (number 669).

2.2. Evaluation of Clinicopathologic Features. We investigated the incidence of metachronous tumors in 357 patients using the Kaplan-Meier method and retrospectively investigated the clinicopathologic features associated with metachronous tumors, including patient age and gender, tumor size, location, gross type, extension of gastric mucosal atrophy, presence of synchronous multiple tumors, histology, and depth. We also evaluated the outcomes of metachronous tumors after ESD.

In patients with synchronous multiple tumors, we chose as the main lesion a tumor that had the highest malignant potential as determined by a malignancy, diffuse type, or increased size or depth. Tumor location and macroscopic types of gastric tumors were classified according to the Japanese Classification of Gastric Carcinoma (JCGC) [18]. In this study, type 0-I (protruded) and type 0-IIa (superficial elevated) were grouped together as "elevated," while type 0IIc (superficial depressed) and type 0-IIa+IIc (elevated with central depression) were grouped together as "depressed." Endoscopic evaluations of atrophic gastritis were determined according to the criteria of the Kimura and Takemoto classification [19]. The pathological diagnosis of each tumor was also judged according to the JCGC criteria [18]. In this study, we included adenoma among the intestinal-type tumors.

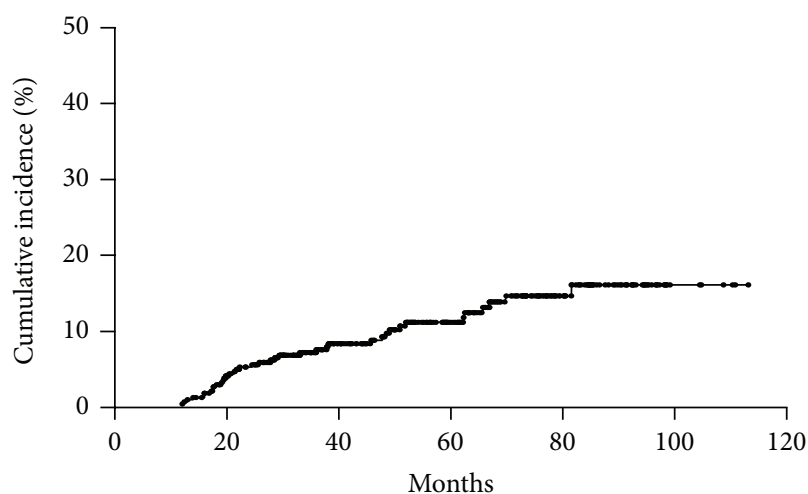

Number at risk

$\begin{array}{lllllll}357 & 337 & 224 & 150 & 65 & 6 & 0\end{array}$

FIgURE 1: Kaplan-Meier curve of the cumulative incidence of metachronous tumors after ESD for gastric tumors.

2.3. Evaluation of Serum Markers. We evaluated the levels of serum gastrin (Gastrin RIA Kit II; Dainabot Co., Ltd., Osaka, Japan) and serum pepsinogen (LZ test; Eiken Chemical Co., Ltd., Tokyo, Japan) instead of performing histological evaluation of the gastric mucosa. We could evaluate fasting serum gastrin and pepsinogen levels in 281 of the 357 patients.

2.4. Statistics. The cumulative incidence of metachronous gastric tumors was evaluated using the Kaplan-Meier method. To analyze potential risk factors for metachronous tumors, we performed univariate analysis using the KaplanMeier method, log-rank test, and Cox's proportional hazards modeling. A $P$ value of $<0.05$ was considered significant. The JMP statistical software package (SAS Institute Inc., Cary, NC, USA) was used for all calculations.

\section{Results}

3.1. Kaplan-Meier Analysis of the Cumulative Incidence of Metachronous Gastric Tumors. We investigated the incidence of metachronous gastric tumors after ESD in 357 patients with gastric tumors using the Kaplan-Meier method (Figure 1). Thirty-nine patients developed metachronous tumors (24 EGCs and 15 adenomas), and the median period until discovery after initial ESD was 26.0 months (range: 12.2-81.8 months). According to the investigation of initial/metachronous tumor, 5 patients had adenoma/adenoma, 2 patients had adenoma/adenocarcinoma, 10 patients had adenocarcinoma/adenoma, and 22 patients had adenocarcinoma/adenocarcinoma, respectively. The cumulative incidence curve of metachronous gastric tumors revealed a gradual increase and an incidence of $2.4 \%$ per year. When we excluded cases in which the initial or second tumors were adenoma from the 357 patients, the incidence of metachronous EGC was $2.0 \%$ per year $(n=236$, data not shown). There was no difference in the incidence of metachronous gastric tumor between adenoma and adenocarcinoma of initial treatment. 
3.2. Clinicopathologic Characteristics of Metachronous Gastric Tumors after ESD. We investigated the clinicopathologic characteristics of the tumors and patients at the time of second tumor discovery in the above-mentioned 39 patients who developed metachronous tumors (Table 1). The average age was 70.3 years, and $35(90 \%)$ of the patients were male. The average tumor size was $11.1 \mathrm{~mm}$ (range: $3-20 \mathrm{~mm}$ ) in diameter. Of 39 lesions, 10 (26\%), 12 (31\%), and 17 (44\%) lesions developed in the upper, middle, and lower third of the stomach, respectively. When we compared the second tumors and initial tumors with regard to development location, the second tumors more frequently developed in the upper third of the stomach $(P=0.0002)$. Eighteen $(46 \%)$ lesions were diagnosed as elevated type and the others were of depressed type. Almost all patients had severe gastric mucosal atrophy, which is termed as open-type according to the Kimura-Takemoto classification. Only $3(8 \%)$ patients had developed multiple tumors at the time of second tumor detection. According to the pathological evaluation, 37 (95\%) patients developed intestinal-type tumors and the others developed diffuse-type tumors. Five cases (13\%) developed submucosal invasive gastric cancers. No advanced gastric cancers occurred. All intramucosal tumors were curatively resected by ESD. Four patients with submucosal gastric cancers underwent additional resection of the stomach, and 1 patient was followed up without surgery. There were no gastric cancer deaths during follow-up period.

\subsection{Analysis of Risk Factors for Metachronous Gastric Tumors} after ESD. According to the univariate analysis performed using the Kaplan-Meier method and log-rank test, only gender significantly affected the incidence of metachronous tumors. The incidence of metachronous tumors was greater among male patients than among female patients $(P=0.04$, Figure 2). As shown in Table 2, the hazard ratio of female to male patients was 0.36 (95\% confidence interval: 0.11-0.89), and no other factors affected the incidence in the univariate analysis according to Cox's proportional hazards model.

\section{Discussion}

In Japan, ESD has been standardized as a local treatment for EGC with no risk of lymph node (LN) metastasis. According to the Japanese gastric cancer treatment guidelines [17], ESD is indicated as a standard treatment for differentiated-type adenocarcinomas without ulcerative findings $(\mathrm{UL}(+))$, with a depth of invasion clinically diagnosed as Tla and a diameter of $\leq 2 \mathrm{~cm}$ (absolute indication). Tumors that are clinically diagnosed as Tla and are (a) of the differentiated type, UL(-), but $>2 \mathrm{~cm}$ in diameter; (b) of the differentiated type, UL $(+)$, and $\leq 3 \mathrm{~cm}$ in diameter; or (c) of the undifferentiated type, $\mathrm{UL}(-)$, and $\leq 2 \mathrm{~cm}$ in diameter have a very low possibility of LN metastasis, and ESD for these tumors is regarded as an investigational treatment (expanded indication). Additionally, resection of differentiated-type adenocarcinomas with submucosal invasion of $<500 \mu \mathrm{m}$ and a diameter of $\leq 3 \mathrm{~cm}$ is considered curative. Some reports have supported the validity of these indications [20-22]. Furthermore, risk factors for
TABLE 1: Clinicopathologic characteristics associated with metachronous gastric tumors.

\begin{tabular}{cc}
\hline Factors & No. of patients \\
\hline Total patients & 39 \\
Age (years) & \\
Mean (range) & $70.3(50-88)$ \\
Gender & \\
Male & $35(90 \%)$ \\
Female & $4(10 \%)$ \\
Tumor size (mm) & \\
Mean (range) & $11.1(3-20)$ \\
Location & \\
Upper third & $10(26 \%)$ \\
Middle third & $12(31 \%)$ \\
Lower third & $17(44 \%)$ \\
Gross type & \\
Elevated & $18(46 \%)$ \\
Depressed & $21(54 \%)$ \\
Gastric mucosal atrophy & \\
Closed & $1(3 \%)$ \\
Open & $38(97 \%)$ \\
Synchronous tumor & $34(87 \%)$ \\
Negative & $3(13 \%)$ \\
Positive & $3(8 \%)$ \\
Histology & \\
Intestinal & \\
Diffuse & \\
Depth & \\
Mucosa & \\
Submucosa & \\
\hline
\end{tabular}

${ }^{\dagger}$ Elevated: 0-I and 0-IIa; depressed: 0-IIc and 0-IIa+IIc.

LN metastasis of submucosal invasive gastric cancer or undifferentiated type EGC have been reported [23-29]. We have been able to perform resection in difficult-to-treat cases such as those with ulceration because of advances in the ESD technique and device [30,31]. ESD may have a potential that the criteria for curative endoscopic resection (ER) is increasingly expanded in the future. It is commonly known that gastric cancers often recur metachronously, and the risk of metachronous multiple tumors after ESD is thought to be higher than that after gastrectomy [7, 13-15].

Our data revealed that the annual incidence of metachronous gastric tumors was $2.4 \%$, which is almost equal to the previously reported incidence $[7,14,15]$. The median interval period to the detection of a second tumor after initial ESD was 26.0 months (range: 12.2-81.8 months), and Kaplan-Meier curve seemed to reach a plateau after 80 months. Kobayashi et al. [32] reported that the median interval between the discovery of metachronous cancer and the initial ER was 3.2 years in patients who were followed up for 3.0 to 19.6 years (median: 5.0 years), and no metachronous cancers were detected in patients who were followed up for more than 10 years. These data suggest that metachronous 


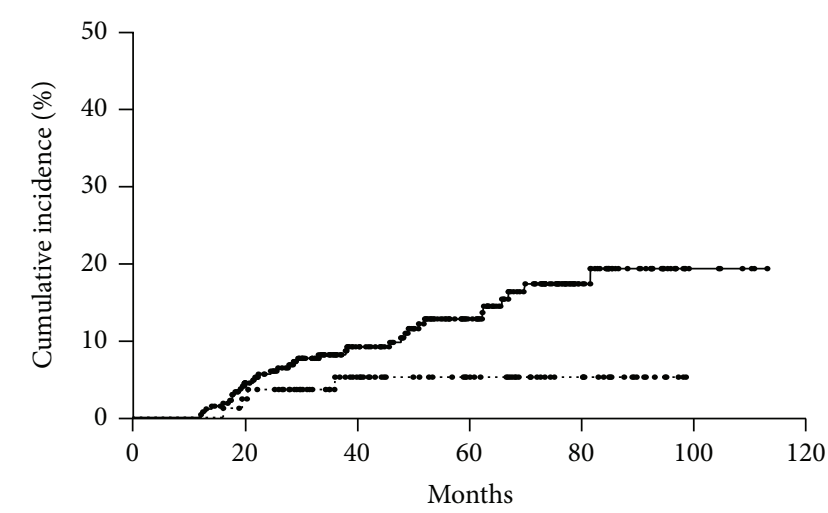

Number at risk

$\begin{array}{lccccccc}\text { Male } & 273 & 256 & 169 & 113 & 45 & 6 & 0 \\ \text { Female } & 84 & 81 & 55 & 37 & 20 & 0 & 0\end{array}$

FIGURE 2: Cumulative incidence of metachronous tumors after ESD in male and female patients. The incidence of metachronous tumors was significantly greater among male patients (solid line) than among female patients (dotted line; $P=0.04$ ).

gastric tumors may develop around 3 years after ESD and that the incidence may gradually decrease after ESD. However, it is necessary to investigate more cases because of too few patients followed up during long term. Since the annual incidence of gastric cancer in $\mathrm{H}$. pylori-positive patients was reported to be $0.38-0.5 \%[4,33]$, after ESD, patients have a higher risk of developing gastric cancer.

We investigated the clinicopathologic characteristics of metachronous gastric tumors and revealed that second lesions tended to develop in the upper third of the stomach. Kato et al. [15] reported that many synchronous gastric cancers after ESD that had been missed by the preoperative endoscopic examination were located in the upper third of the stomach. Since it is difficult to detect tumors in this region, we might be able to detect them by more frequent and careful endoscopic examinations after ESD.

Our data showed that only gender significantly affected the metachronous tumor incidence. Some reports indicated that male patients more frequently developed metachronous gastric cancer after surgery or ER [13, 15, 32]. It is commonly known that the incidence of gastric cancer is higher in male than in female. It has also been reported that differences in smoking rates and salt intake between male and female affect the incidence $[34,35]$. Patient age and the presence of synchronous multiple gastric cancers at the time of the initial ER have been reported to significantly affect the incidence of metachronous gastric cancer [36], and antral atrophy was significantly associated with incidence in a previous multivariate analysis [14]. However, these factors did not significantly affect the results of this study. The fact that patients with synchronous tumors are susceptible to metachronous tumors implies that gene mutations or gastric mucosal conditions may be causes of metachronous tumors. A few reports indicated that microsatellite instability (MSI) was a factor that affected the development of both synchronous and metachronous multiple gastric cancers, and
TABLE 2: Analysis of risk factors for metachronous gastric tumors according to Cox's proportional hazards model.

\begin{tabular}{|c|c|c|c|}
\hline \multirow{2}{*}{ Factors } & \multirow{2}{*}{ No. } & \multicolumn{2}{|c|}{ Univariate analysis } \\
\hline & & Hazard ratio & $95 \% \mathrm{CI}$ \\
\hline Age (1-year increment) & & 1.00 & $0.97-1.04$ \\
\hline \multicolumn{4}{|l|}{ Gender } \\
\hline Male & 272 & 1 & \\
\hline Female & 85 & 0.36 & $0.11-0.89$ \\
\hline Tumor size (increment of $1 \mathrm{~mm}$ ) & & 1.01 & $0.98-1.03$ \\
\hline \multicolumn{4}{|l|}{ Location } \\
\hline Upper third & 38 & 1 & \\
\hline Middle third & 91 & 1.68 & $0.55-7.29$ \\
\hline Lower third & 228 & 1.02 & $0.35-4.32$ \\
\hline \multicolumn{4}{|l|}{ Gross type } \\
\hline Elevated & 161 & 1 & \\
\hline Depressed & 196 & 1.07 & $0.57-2.04$ \\
\hline \multicolumn{4}{|l|}{ Gastric mucosal atrophy } \\
\hline Closed & 30 & 1 & \\
\hline Open & 327 & 1.20 & $0.43-4.99$ \\
\hline \multicolumn{4}{|l|}{ Synchronous tumor } \\
\hline Negative & 307 & 1 & \\
\hline Positive & 50 & 1.26 & $0.47-2.79$ \\
\hline \multicolumn{4}{|l|}{ Histology } \\
\hline Intestinal & 331 & 1 & \\
\hline Diffuse & 26 & 0.84 & $0.20-2.33$ \\
\hline \multicolumn{4}{|l|}{ Depth } \\
\hline Mucosa & 326 & 1 & \\
\hline Submucosa & 31 & 1.05 & $0.25-2.92$ \\
\hline \multicolumn{4}{|l|}{ Serum gastrin } \\
\hline$\leq 100 \mathrm{pg} / \mathrm{mL}$ & 94 & 1 & \\
\hline$>100 \mathrm{pg} / \mathrm{mL}$ & 187 & 0.58 & $0.28-1.21$ \\
\hline \multicolumn{4}{|l|}{ Serum pepsinogen $^{\dagger}$} \\
\hline Negative & 111 & 1 & \\
\hline Positive & 170 & 1.23 & $0.59-2.75$ \\
\hline
\end{tabular}

CI: confidence interval.

${ }^{\dagger}$ Serum pepsinogen-positive: $\mathrm{PG} \mathrm{I} \leq 70 \mathrm{ng} / \mathrm{mL}$ and $\mathrm{PG} \mathrm{I} / \mathrm{II} \leq 3$.

the frequency of MSI was found to be significantly higher in patients with metachronous gastric cancers than in those with single gastric cancers $[37,38]$. Although we could not evaluate the histological condition of the gastric mucosa, we investigated serum gastrin and pepsinogen levels instead. Serum gastrin levels of patients with severe gastric mucosal atrophy are higher than those of patients with mild or no gastric mucosal atrophy because severe atrophy reduces the secretion of gastric acid [39, 40]. Serum levels of pepsinogen (PG) I and PG II and the PG I/II ratio vary according to gastric mucosal atrophy and inflammation [40-42]. Cases with PG $\mathrm{I} \leq 70 \mathrm{ng} / \mathrm{mL}$ and $\mathrm{PG} \mathrm{I} / \mathrm{II} \leq 3$ were regarded as PG positive, indicative of gastric mucosal atrophy [41]. It was thought that gastric mucosal condition seldom affected the metachronous tumor incidence because serum gastrin and pepsinogen levels did not affect the incidence. In this study, we excluded patients who received $H$. pylori eradication therapy to avoid the influence of $H$. pylori eradication on the development of 
metachronous tumor. Eradication of $H$. pylori infection was reported to reduce the risk of gastric cancer development $[33,43]$. Recently, an open-label, randomized controlled trial showed that $H$. pylori eradication prevented the development of metachronous cancer after ER for EGC patients during a 3-year follow-up period [7], and therefore, eradication therapy is recommended after ER for EGC in Japan [44]. As a result, metachronous gastric cancer detection after $H$. pylori eradication will increase. Furthermore, some reports suggested that macroscopic/biological features of gastric tumors could change after $H$. pylori eradication [45-47]. In the near future, it will be necessary to investigate predictive factors of metachronous gastric tumors after ESD for gastric tumors in patients who have undergone $H$. pylori eradication.

\section{Conclusions}

Patients with a previous history of gastric tumors have an increased risk of subsequent gastric tumor development and male patients should be carefully followed up after ESD for gastric tumor.

\section{Conflict of Interests}

No author has conflict of interests or financial arrangements that could potentially influence the described research.

\section{References}

[1] M. P. Curado, B. Edwards, H. R. Shin et al., Cancer Incidence in Five Continents, vol. 9, IARC Scientific Publication, Lyon, France, 2009.

[2] J. Parsonnet, G. D. Friedman, D. P. Vandersteen et al., "Helicobacter pylori infection and the risk of gastric carcinoma," The New England Journal of Medicine, vol. 325, no. 16, pp. 1127-1131, 1991.

[3] A. Nomura, G. N. Stemmermann, P.-H. Chyou, I. Kato, G. I. Perez-Perez, and M. J. Blaser, "Helicobacter pylori infection and gastric carcinoma among Japanese Americans in Hawaii," The New England Journal of Medicine, vol. 325, no. 16, pp. 1132-1136, 1991.

[4] N. Uemura, S. Okamoto, S. Yamamoto et al., "Helicobacter pylori infection and the development of gastric cancer," The New England Journal of Medicine, vol. 345, no. 11, pp. 784-789, 2001.

[5] T. Nakajima, I. Oda, T. Gotoda et al., "Metachronous gastric cancers after endoscopic resection: how effective is annual endoscopic surveillance?" Gastric Cancer, vol. 9, no. 2, pp. 9398, 2006 .

[6] J. Nasu, T. Doi, H. Endo, T. Nishina, S. Hirasaki, and I. Hyodo, "Characteristics of metachronous multiple early gastric cancers after endoscopic mucosal resection," Endoscopy, vol. 37, no. 10, pp. 990-993, 2005.

[7] K. Fukase, M. Kato, S. Kikuchi et al., "Effect of eradication of Helicobacter pylori on incidence of metachronous gastric carcinoma after endoscopic resection of early gastric cancer: an open-label, randomised controlled trial," The Lancet, vol. 372, no. 9636, pp. 392-397, 2008.

[8] B. C. Wong, S. K. Lam, W. M. Wong et al., "Helicobacter pylori eradication to prevent gastric cancer in a high-risk region of China: a randomized controlled trial," The Journal of the American Medical Association, vol. 291, no. 2, pp. 187-194, 2004.

[9] S. Oka, S. Tanaka, I. Kaneko et al., "Advantage of endoscopic submucosal dissection compared with EMR for early gastric cancer," Gastrointestinal Endoscopy, vol. 64, no. 6, pp. 877-883, 2006.

[10] T. Gotoda, H. Yamamoto, and R. M. Soetikno, "Endoscopic submucosal dissection of early gastric cancer," Journal of Gastroenterology, vol. 41, no. 10, pp. 929-942, 2006.

[11] A. Imagawa, H. Okada, Y. Kawahara et al., "Endoscopic submucosal dissection for early gastric cancer: results and degrees of technical difficulty as well as sucsess," Endoscopy, vol. 38, no. 10, pp. 987-990, 2006.

[12] I. Oda, D. Saito, M. Tada et al., "A multicenter retrospective study of endoscopic resection for early gastric cancer," Gastric Cancer, vol. 9, no. 4, pp. 262-270, 2006.

[13] I. Nozaki, J. Nasu, Y. Kubo, M. Tanada, R. Nishimura, and A. Kurita, "Risk factors for metachronous gastric cancer in the remnant stomach after early cancer surgery," World Journal of Surgery, vol. 34, no. 7, pp. 1548-1554, 2010.

[14] J. S. Han, J. S. Jang, S. R. Choi et al., "A study of metachronous cancer after endoscopic resection of early gastric cancer," Scandinavian Journal of Gastroenterology, vol. 46, no. 9, pp. 1099-1104, 2011.

[15] M. Kato, T. Nishida, K. Yamamoto et al., "Scheduled endoscopic surveillance controls secondary cancer after curative endoscopic resection for early gastric cancer: a multicentre retrospective cohort study by Osaka University ESD study group," Gut, vol. 62, no. 10, pp. 1425-1432, 2013.

[16] T. Kitoh, H. Yanai, Y. Matsubara et al., "Endoscopic findings potentially predictive of gastric cancer in borderline lesions diagnosed by forceps biopsy," Hepato-Gastroenterology, vol. 52, no. 62, pp. 404-408, 2005.

[17] Japanese Gastric Cancer Association, "Japanese gastric cancer treatment guidelines 2010 (ver. 3)," Gastric Cancer, vol. 14, no. 2, pp. 113-123, 2011.

[18] Japanese Gastric Cancer Association, "Japanese classification of gastric carcinoma: 3rd english edition," Gastric Cancer, vol. 14, no. 2, pp. 101-112, 2011.

[19] K. Kimura and T. Takemoto, "An endoscopic recognition of the atrophic border and its significance in chronic gastritis," Endoscopy, vol. 1, no. 3, pp. 87-97, 1969.

[20] T. Gotoda, A. Yanagisawa, M. Sasako et al., "Incidence of lymph node metastasis from early gastric cancer: estimation with a large number of cases at two large centers," Gastric Cancer, vol. 3, no. 4, pp. 219-225, 2000.

[21] T. Gotoda, M. Iwasaki, C. Kusano, S. Seewald, and I. Oda, "Endoscopie resection of early gastric cancer treated by guideline and expanded National Cancer Centre criteria," British Journal of Surgery, vol. 97, no. 6, pp. 868-871, 2010.

[22] Y. Sanomura, S. Oka, S. Tanaka et al., "Clinical validity of endoscopic submucosal dissection for submucosal invasive gastric cancer: a single-center study," Gastric Cancer, vol. 15, no. 1, pp. 97-105, 2012.

[23] Y. Sanomura, S. Oka, S. Tanaka et al., "Predicting the absence of lymph node metastasis of submucosal invasive gastric cancer: expansion of the criteria for curative endoscopic resection," Scandinavian Journal of Gastroenterology, vol. 45, no. 12, pp. 1480-1487, 2010.

[24] M. S. Lim, H.-W. Lee, H. Im et al., "Predictable factors for lymph node metastasis in early gastric cancer-analysis of single 
institutional experience," Journal of Gastrointestinal Surgery, vol. 15, no. 10, pp. 1783-1788, 2011.

[25] M. Fujii, Y. Egashira, H. Akutagawa et al., "Pathological factors related to lymph node metastasis of submucosally invasive gastric cancer: criteria for additional gastrectomy after endoscopic resection," Gastric Cancer, vol. 16, no. 4, pp. 521-530, 2013.

[26] T. Hirasawa, T. Gotoda, S. Miyata et al., "Incidence of lymph node metastasis and the feasibility of endoscopic resection for undifferentiated-type early gastric cancer," Gastric Cancer, vol. 12, no. 3, pp. 148-152, 2009.

[27] N. Hanaoka, S. Tanabe, T. Mikami, I. Okayasu, and K. Saigenji, "Mixed-histologic-type submucosal invasive gastric cancer as a risk factor for lymph node metastasis: feasibility of endoscopic submucosal dissection," Endoscopy, vol. 41, no. 5, pp. 427-432, 2009.

[28] C. Kunisaki, M. Takahashi, Y. Nagahori et al., "Risk factors for lymph node metastasis in histologically poorly differentiated type early gastric cancer," Endoscopy, vol. 41, no. 6, pp. 498-503, 2009.

[29] J.-H. Tong, Z. Sun, Z.-N. Wang et al., "Early gastric cancer with signet-ring cell histologic type: risk factors of lymph node metastasis and indications of endoscopic surgery," Surgery, vol. 149, no. 3, pp. 356-363, 2011.

[30] M. Higashimaya, S. Oka, S. Tanaka et al., "Outcome of endoscopic submucosal dissection for gastric neoplasm in relationship to endoscopic classification of submucosal fibrosis," Gastric Cancer, vol. 16, no. 3, pp. 404-410, 2013.

[31] M. Higashimaya, S. Oka, S. Tanaka et al., "Endoscopic submucosal dissection for residual early gastric cancer after endoscopic submucosal dissection," Gastrointestinal Endoscopy, vol. 77, no. 2, pp. 298-302, 2013.

[32] M. Kobayashi, R. Narisawa, Y. Sato, M. Takeuchi, and Y. Aoyagi, "Self-limiting risk of metachronous gastric cancers after endoscopic resection," Digestive Endoscopy, vol. 22, no. 3, pp. 169-173, 2010.

[33] M. Kato, M. Asaka, T. Nakamura et al., "Helicobacter pylori eradication prevents the development of gastric cancer," Alimentary Pharmacology \& Therapeutics Symposium Series, vol. 2, no. 1, pp. 203-206, 2006.

[34] Y. Nishino, M. Inoue, I. Tsuji et al., "Tobacco smoking and gastric cancer risk: an evaluation based on a systematic review of epidemiologic evidence among the Japanese population," Japanese Journal of Clinical Oncology, vol. 36, no. 12, pp. 800$807,2006$.

[35] S. Tsugane, S. Sasazuki, M. Kobayashi, and S. Sasaki, "Salt and salted food intake and subsequent risk of gastric cancer among middle-aged Japanese men and women," British Journal of Cancer, vol. 90, no. 1, pp. 128-134, 2004.

[36] N. Arima, K. Adachi, T. Katsube et al., "Predictive factors for metachronous recurrence of early gastric cancer after endoscopic treatment," Journal of Clinical Gastroenterology, vol. 29, no. 1, pp. 44-47, 1999.

[37] E. Miyoshi, K. Haruma, T. Hiyama et al., "Microsatellite instability is a genetic marker for the development of multiple gastric cancers," International Journal of Cancer, vol. 95, no. 6, pp. 350353, 2001.

[38] S. H. Kim, B. K. Ahn, Y. S. Nam, J. Y. Pyo, Y. H. Oh, and K. H. Lee, "Microsatellite instability is associated with the clinicopathologic features of gastric cancer in sporadic gastric cancer patients," Journal of Gastric Cancer, vol. 10, no. 4, pp. 149$154,2010$.
[39] P. Sipponen, J. Valle, K. Varis, M. Kekki, T. Ihamäki, and M. Siurala, "Fasting levels of serum gastrin in different functional and morphologic states of the antrofundal mucosa: an analysis of 860 subjects," Scandinavian Journal of Gastroenterology, vol. 25, no. 5, pp. 513-519, 1990.

[40] B. Germaná, F. di Mario, L. G. Cavallaro et al., "Clinical usefulness of serum pepsinogens I and II, gastrin-17 and antiHelicobacter pylori antibodies in the management of dyspeptic patients in primary care," Digestive and Liver Disease, vol. 37, no. 7, pp. 501-508, 2005.

[41] K. Miki, M. Ichinose, and A. Shimizu, "Serum pepsinogens as a screening test of extensive chronic gastritis," Gastroenterologia Japonica, vol. 22, no. 2, pp. 133-141, 1987.

[42] K. Kiyohira, M. Yoshihara, M. Ito, K. Haruma, S. Tanaka, and K. Chayama, "Serum pepsinogen concentration as a marker of Helicobacter pylori infection and the histologic grade of gastritis; evaluation of gastric mucosa by serum pepsinogen levels," Journal of Gastroenterology, vol. 38, no. 4, pp. 332-338, 2003.

[43] N. Uemura, T. Mukai, S. Okamoto et al., "Effect of Helicobacter pylori eradication on subsequent development of cancer after endoscopic resection of early gastric cancer," Cancer Epidemiology, Biomarkers \& Prevention, vol. 6, no. 8, pp. 639-642, 1997.

[44] M. Asaka, M. Kato, S.-I. Takahashi et al., "Guidelines for the management of Helicobacter pylori infection in Japan: 2009 revised edition," Helicobacter, vol. 15, no. 1, pp. 1-20, 2010.

[45] M. Ito, S. Tanaka, S. Takata et al., "Morphological changes in human gastric tumours after eradication therapy of Helicobacter pylori in a short-term follow-up," Alimentary Pharmacology \& Therapeutics, vol. 21, no. 5, pp. 559-566, 2005.

[46] S. Takata, M. Ito, M. Yoshihara et al., "Host factors contributing to the discovery of gastric cancer after successful eradication therapy of Helicobacter pylori: preliminary report," Journal of Gastroenterology and Hepatology, vol. 22, no. 4, pp. 571-576, 2007.

[47] T. Matsuo, M. Ito, M. Tatsugami et al., "Gastric cancer development after Helicobacter pylori eradication therapy: a new form of gastric neoplasia," Digestion, vol. 85, no. 1, pp. 61-67, 2012. 


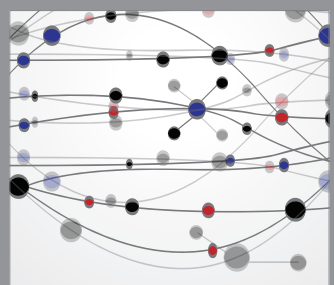

The Scientific World Journal
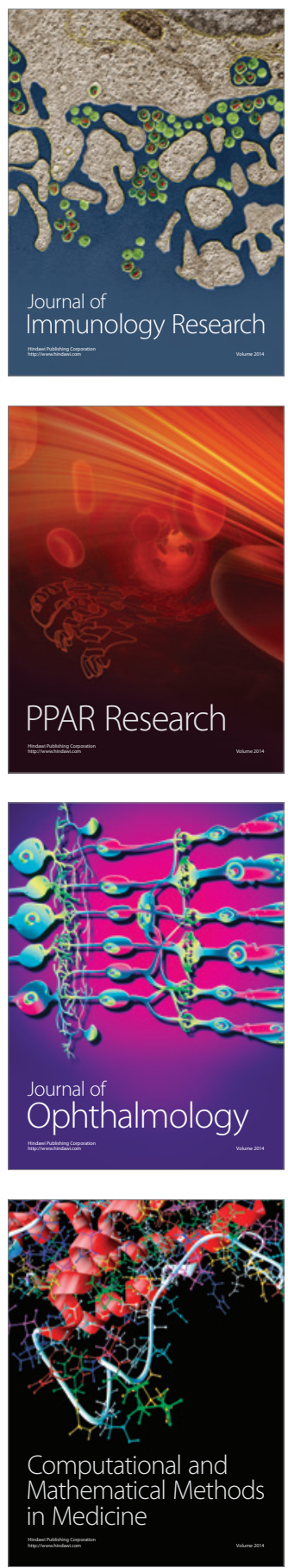

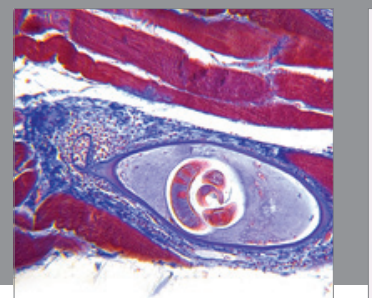

Gastroenterology

Research and Practice
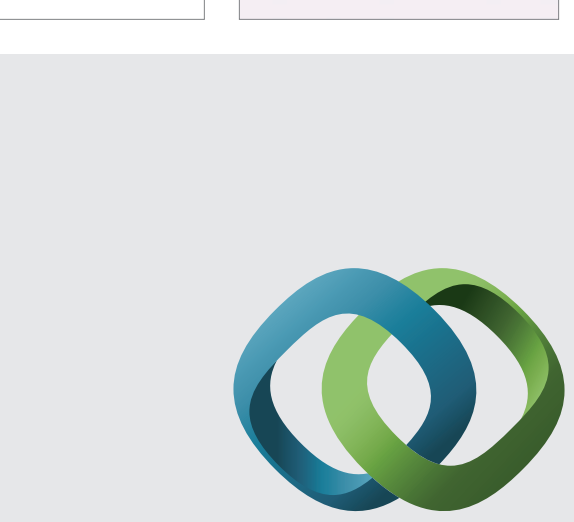

\section{Hindawi}

Submit your manuscripts at

http://www.hindawi.com
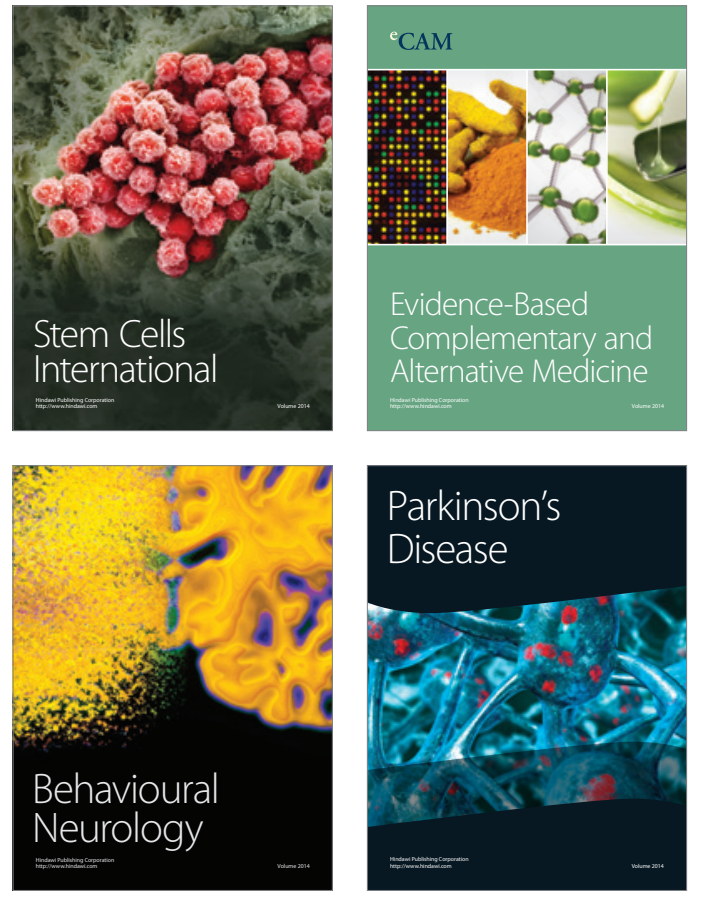
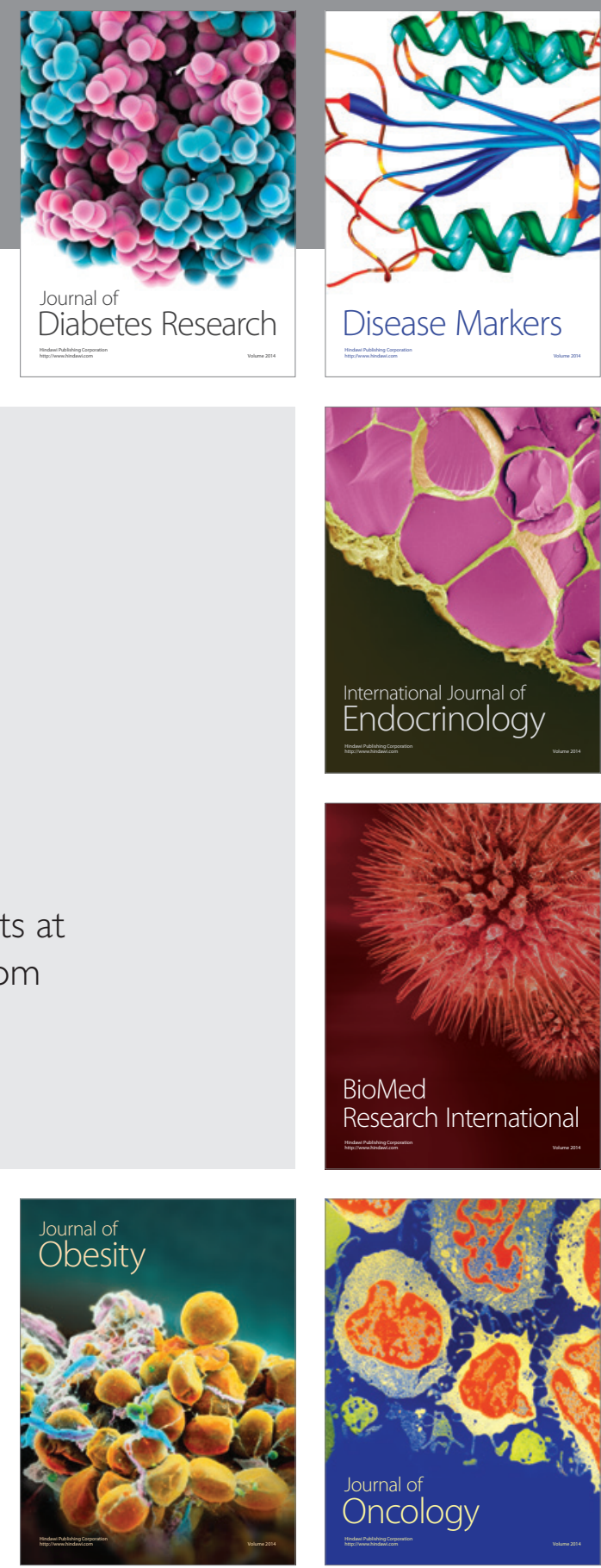

Disease Markers
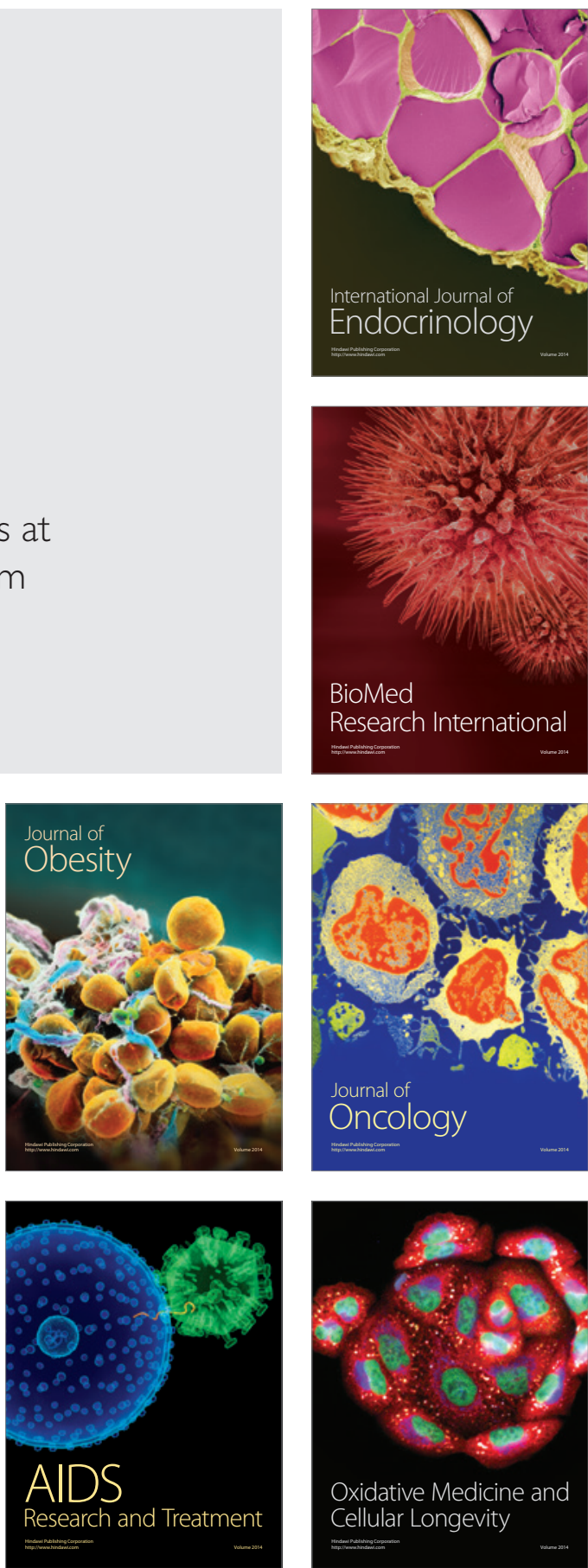